\title{
A CONVERGENCE CRITERION FOR FOURIER SERIES
}

\author{
M. TOMIC
}

1.1. It is known that the condition

$$
\omega\left(x_{0}, h\right) \equiv f\left(x_{0}+h\right)-f\left(x_{0}\right)=o\left(\frac{1}{|\log h|}\right), \quad h \rightarrow 0,
$$

for a special $x_{0}$ does not imply convergence of the Fourier series $s[f]$ of $f(x)$ for that $x_{0}$. The Hardy-Littlewood convergence test [4, p. 63] gives the convergence of $S[f]$ at $x_{0}$, provided that the condition (1) is satisfied, and the coefficients of $S[f], a_{n}$ and $b_{n}$ are $O\left(n^{-\theta}\right)$ for some positive $\theta$.

The aim of the note is to give a sufficient condition on the function $\omega\left(x_{0}, h\right)$ which will insure the convergence of the $S[f]$ at the point $x_{0}$. A similar condition was given by the author [3] under some additional assumptions. The first object of this note is to give a direct proof for this result.

Following A. Zygmund [4, p. 186] we shall use the following definition: A positive function $L(t)$ defined for $0<t<\epsilon$ will be called slowly varying at the point $t=0$, if, for any $\delta>0, L(t) t^{\delta}$ is ultimately a decreasing and $L(t) t^{-\delta}$ increasing function of $t$ for $t \rightarrow 0$. From this definition we deduce immediately: If $L(t)$ is slowly varying, then

$$
\frac{L(\lambda t)}{L(\lambda)} \rightarrow 1, \quad \text { as } \lambda \rightarrow 0,
$$

for every fixed $t>0$, and even uniformly in every interval $a \leqq t \leqq b$, $a>0, b<\infty$.

Let $f(x)$ be a periodic function with period $2 \pi$ and $L$-integrable over $[-\pi, \pi], s_{n}(x) n$th partial sum of its Fourier series. In $\$ 2.1$ we shall prove the following theorem.

THEOREM I. If at the point $x_{0}$

$$
f\left(x_{0}+t\right)+f\left(x_{0}-t\right)-2 f\left(x_{0}\right)=\phi\left(x_{0}, t\right)=\phi(t) \rightarrow 0, \quad t \rightarrow 0,
$$

and for $t \rightarrow 0, \phi(t) \equiv L(t)$ is slowly varying then $s_{n}\left(x_{0}\right) \rightarrow f\left(x_{0}\right), n \rightarrow \infty$.

1.2. Let $f(x)$ be a continuous function of period $2 \pi$. It is known that $s_{n}(x)-f(x)=O\left(n^{-\alpha} \log n\right)$ uniformly in $x$ if $f(x)$ belongs to Lip $\alpha$, i.e., if

Received by the editors March 4, 1963. 


$$
\sup _{0<|h| \leqq \delta}|f(x+h)-f(x)|=O\left(\delta^{\alpha}\right), \quad 0<\alpha \leqq 1 .
$$

The factor $\log n$ cannot be omitted even under the additional condition that $f$ is of bounded variation [2]. Following Salem and Zygmund $[2 ; 4$, p. 64] the function $f(x)$ will be called of monotonic type if $f(x)+C x$ is monotonic for suitable constant $C$. Then, if $f$ is of monotonic type and belongs to Lip $\alpha, 0<\alpha<1, s_{n}(x)-f(x)=O\left(n^{-\alpha}\right)$ uniformly in $x$. In $\$ 2.2$ we shall prove the following extension of the theorem of Salem and Zygmund.

THEOREM II. Let $f(x)$ be a continuous function over $[-\pi, \pi]$ and of monotonic type, with

$$
\omega(x, t)=\omega(t)=\sup _{0<|h| \leqq t}|f(x+h)-f(x)|=L(t), t \rightarrow 0, x \in[-\pi, \pi] ;
$$

then

$$
\left|s_{n}(x)-f(x)\right|=O\left(L\left(\frac{\pi}{n}\right)\right)=O\left(\omega\left(\frac{\pi}{n}\right)\right) .
$$

2.1. Proof of Theorem I. Without loss of generality, we may suppose that $x_{0}=0, f$ is even, and $f(0)=0$. Using the known formula for partial sum $s_{n}(x)$ of the Fourier series $S[f][4$, p. 55] we have

$$
s_{n}(0)=\frac{2}{\pi} \int_{0}^{e} f(t) \frac{\sin n t}{t} d t+o(1) .
$$

From the fact that $f(t)$ is a slowly varying function in the neighbourhood of $t=0$, (3) can be written as

$$
\begin{aligned}
\frac{2}{\pi} \int_{0}^{e} f(t) \frac{\sin n t}{t} d t & =\frac{2}{\pi} \int_{0}^{\phi(n)} L(t) \frac{\sin n t}{t} d t+\frac{2}{\pi} \int_{\phi(n)}^{e} f(t) \frac{\sin n t}{t} d t \\
& =J_{1}+J_{2},
\end{aligned}
$$

where $\phi(n)=\sqrt{ }\left(\omega_{1}(\pi / n)\right) \rightarrow 0, n \rightarrow \infty$ and $\omega_{1}(\delta)=\omega_{1}(\delta, f)$ denotes the integral modulus of continuity of $f$, i.e.,

$$
\omega_{1}(\delta, f)=\sup _{0<|h| \leqq \delta} \int_{-\pi}^{\pi}|f(t+h)-f(t)| d t .
$$

Take $n t=\tau, n^{-1}=\lambda, n \phi(n)=\Lambda$ and we have for $J_{1}$

$$
(\pi / 2) J_{1}=\int_{0}^{\Delta} L(\lambda t) \frac{\sin t}{t} d t .
$$


We split $J_{1}$ into three parts ${ }^{1}$

$$
\begin{aligned}
(\pi / 2) J_{1}= & \int_{0}^{\delta} L(\lambda t) \frac{\sin t}{t} d t+\int_{\Delta}^{\Delta} L(\lambda t) \frac{\sin t}{t} d t \\
& +L(\lambda) \int_{\delta}^{\Delta}\left[\frac{L(\lambda t)}{L(\lambda)}-1\right] \frac{\sin t}{t} d t+L(\lambda) \int_{\delta}^{\Delta} \frac{\sin t}{t} d t \\
= & I_{1}+I_{2}+I_{3}+K L(\lambda),
\end{aligned}
$$

with arbitrary but fixed $\delta$ and $\Delta$ and $K=K(\delta, \Delta)=\int_{\delta}^{\Delta} t^{-1} \sin t d t$ for every $\delta$ and $\Delta$. Assuming that $0<\eta<1$, we have

$$
I_{1}=\lambda \rightarrow \int_{0}^{\delta}(\lambda t)^{\eta} L(\lambda t) \frac{\sin t}{t^{1+\eta}} d t
$$

and since $L(t)$ is slowly varying, the last integral can be majorized by

$$
\left|I_{1}\right| \leqq \delta^{\eta} L(\lambda \delta) \int_{0}^{\delta} \frac{|\sin t|}{t^{1+\eta}} d t, \quad 0<\eta<1
$$

and so $I_{1} \rightarrow 0$ for $\lambda \rightarrow 0$.

Similarly with $0<\eta<1$ we obtain

$$
I_{2}=\int_{\Delta}^{\Delta} L(\lambda t) \frac{\sin t}{t} d t=\lambda^{\eta} \int_{\Delta}^{\Delta}(\lambda t)^{-\eta} L(\lambda t) \frac{\sin t}{t^{1-\eta}} d t .
$$

Using the fact that $(\lambda t)^{-\eta} L(\lambda t)$ is a decreasing function in $\Delta<t<\Lambda$, and applying the second mean value theorem, we get

$$
I_{2}=\frac{L(\lambda \Delta)}{\Delta^{\eta}} \int_{\Delta}^{\Lambda_{1}} \frac{\sin t}{t^{1-\eta}} d t, \quad \Delta \leqq \Lambda_{1} \leqq \Lambda,
$$

and the last integral being convergent, we have $I_{2} \rightarrow 0, \lambda \rightarrow 0$.

Finally, since $\delta$ and $\Delta$ are fixed, the passage to the limit $\lambda \rightarrow 0$ under the integral sign in

$$
I_{3}=L(\lambda) \int_{\delta}^{\Delta}\left[\frac{L(\lambda t)}{L(\lambda)}-1\right] \frac{\sin t}{t} d t,
$$

is justified by (2), and we have $I_{3}=o(1)$, and consequently by (5), (6) and $(7) J_{1}=o(1), n \rightarrow \infty$.

Now, consider the integral

$$
(\pi / 2) J_{2}=\int_{\phi(n)}^{e} f(t) \frac{\sin t}{t} d t .
$$

\footnotetext{
1 For a similar decomposition see [1].
} 
Putting $t=\tau+\pi / n$, we have

$$
\begin{aligned}
\pi \cdot J_{2}= & -\int_{\phi(n)-\pi / n}^{\phi(n)} \frac{f(t+\pi / n)}{t+\pi / n} \sin n t d t \\
& +\int_{\phi(n)}^{\epsilon}\left(\frac{f(t)}{t}-\frac{f(t+\pi / n)}{t+\pi / n}\right) \sin n t d t+\int_{\epsilon-\pi / n}^{e} \frac{f(t+\pi / n)}{t+\pi / n} \sin n t d t \\
& =-K_{1}+K_{2}+K_{3}
\end{aligned}
$$

say. In the neighbourhood of $t=0, f(t)$ tends to 0 as $t \rightarrow 0$, and $K_{1}$ can be majorized by

$$
\left|K_{1}\right| \leqq \frac{|f(\phi(n)+\pi / n)|}{\phi(n)} \cdot \frac{\pi}{n}=o\left(\frac{1}{n \phi(n)}\right), \quad n \rightarrow \infty .
$$

Since $\epsilon$ is fixed and the integrand in $K_{3}$ ultimately bounded, we have $K_{3}=o(1), n \rightarrow \infty$. Now, $K_{2}$ may also be written as

$$
\begin{aligned}
K_{2}= & \int_{\phi(n)}^{c} \frac{f(t)-f(t+\pi / n)}{t} \sin n t d t \\
& +\int_{\phi(n)}^{c}\left\{\frac{1}{t}-\frac{1}{t+\pi / n}\right\} f\left(t+\frac{\pi}{n}\right) \sin n t d t \\
= & K_{21}+K_{22} .
\end{aligned}
$$

Applying in the last integral the second mean value theorem to $\{\cdot\}$, we find

$$
\left|K_{22}\right| \leqq \frac{\pi}{n \phi^{2}(n)}\left|\int_{\phi(n)}^{e_{1}} f\left(t+\frac{\pi}{n}\right) \sin n t d t\right|=o\left(\frac{1}{n \phi^{2}(n)}\right) .
$$

Finally,

$$
\begin{aligned}
\left|K_{21}\right| & \leqq \int_{\phi(n)}^{e} \frac{|f(t)-f(t+\pi / n)|}{t} d t \\
& \leqq \frac{1}{\phi(n)} \int_{\phi(n)}^{c}\left|f(t)-f\left(t+\frac{\pi}{n}\right)\right| d t \leqq \frac{1}{\phi(n)} \omega_{1}\left(\frac{\pi}{n}\right) .
\end{aligned}
$$

If we choose $\phi(n)=\sqrt{ }\left(\omega_{1}(\pi / n)\right)$, it follows from (8), (9) and (10) that $\mathfrak{J}_{2}=o(1), n \rightarrow \infty$, and this proves Theorem I.

2.2. Proof of Theorem II. With the same reasoning as in [4, p. 64] we can suppose that $g(x)=f(x)+C x$ is increasing. The difference $s_{n}(x)-f(x)$ is given by

$$
\pi^{-1} \int_{-\pi}^{\pi}(f(x+t)-f(x)) D_{n}(t) d t
$$


where $D_{n}(t)$ denotes the Dirichlet kernel. Replacing $f(x)$ by $g(x)[\mathbf{4}$, p. 64] we obtain for the last integral

$$
\pi^{-1} \int_{-\pi}^{\pi}(g(x+t)-g(x)) D_{n}(t) d t
$$

and we can consider only the integral over $(0, \pi)$. The proof for the remaining part is the same.

Let

$$
J=\pi^{-1} \int_{0}^{\pi}(g(x+t)-g(x)) D_{n}(t) d t=\pi^{-1}\left(\int_{0}^{\phi(n)}+\int_{\phi(n)}^{\epsilon}+\int_{0}^{\pi}\right) .
$$

In the last integral over $(\epsilon, \pi)$ the function

$$
\{g(x+t)-g(x)\}(2 \sin (t / 2))^{-1}
$$

is of bounded variation with respect to $t$, and this integral is therefore $O(1 / n)$. We have

$$
\pi \mathfrak{I}=\int_{0}^{\phi(n)}+\int_{\phi(n)}^{e}+O\left(\frac{1}{n}\right)=J_{1}+J_{2}+O\left(\frac{1}{n}\right) .
$$

For $\epsilon$ sufficiently small but fixed, $\Phi(t)=\left\{(\sin t)^{-1}-t^{-1}\right\}$ is increasing over $\phi(n)<t<\epsilon$ and by the second mean value theorem we have

$$
\begin{aligned}
\int_{\phi(n)}^{e}\{g(x+t) & -g(x)\}\left\{\frac{1}{2 \sin (t / 2)}-\frac{1}{t}\right\} \sin \left(n+\frac{1}{2}\right) t d t \\
& =\Phi(\epsilon) \int_{\xi}^{\epsilon}\{g(x+t)-g(x)\} \sin \left(n+\frac{1}{2}\right) t d t=O\left(\frac{1}{n}\right) .
\end{aligned}
$$

Finally, we can replace here $\sin \left(n+\frac{1}{2}\right) t$ by $\sin n t$ with error $O(1 / n)$, and we have then that $J_{2}$ in (11) is equal to

$$
J_{2}=\int_{\phi(n)}^{e} \frac{g(x+t)-g(x)}{t} \sin n t d t+O\left(\frac{1}{n}\right) .
$$

If we choose $\phi(n) \rightarrow 0$ so that $[n \phi(n)]^{-1}=o(\omega(\pi / n))$ and if we apply the second mean value theorem to $1 / t$ we obtain

$$
\begin{aligned}
J_{2} & =\frac{1}{\phi(n)} \int_{\phi_{1}(n)}^{\epsilon}[g(x+t)-g(x)] \sin n t d t+O\left(\frac{1}{n}\right) \\
& =O\left(\frac{1}{n \phi(n)}\right)=o(\omega(\pi / n)) .
\end{aligned}
$$


Hence, for $t \rightarrow 0, g(x+t)-g(x)=C t+L(t)$ and we have from (11)

$$
J_{1}=\int_{0}^{\phi(n)} \frac{L(t)}{t} \sin n t d t+o\left(\frac{1}{n}\right) .
$$

This integral is of the same type as $J_{1}$ in 2.1 . It can be estimated in the same manner. We divide $J_{1}$ as in (4). Then we can choose $\delta$ small enough, respectively $\Delta$ sufficiently large, so that $I_{1}$ and $I_{3}$ in (5) and (6) are $o(L(\pi / n))$, and making $n \rightarrow \infty$ we have from (4)

$$
J_{1}=L\left(\frac{\pi}{n}\right) \int_{\delta}^{\Delta} \frac{\sin t}{t} d t+o\left(L\left(\frac{\pi}{n}\right)\right) .
$$

From this estimation and (12) we obtain by (11): $J=O(L(\pi / n))$. Thus Theorem II is proved.

\section{REFERENCES}

1. S. Aljančić, R. Bojaniદ et M. Tomić, Sur la valeur asymptotique d'une classe des intégrales définies, Acad. Serbe Sci. Publ. Inst. Math. 7 (1954), 81-94.

2. R. Salem and A. Zygmund, The approximation by partial sums of Fourier series, Trans. Amer. Math. Soc. 59 (1946), 14-22.

3. M. Tomić, Sur un critère pour la convergence de séries de Fourier dans un point fixe, Acad. Serbe Sci. Arts. Glas Cl. Sci. Math. Nat. 22 (1961), 225-232. (Serbian). 1959.

4. A. Zygmund, Trigonometric series, Vol. I, Cambridge Univ. Press, Cambridge,

University of Belgrade, Belgrade, Yugoslavia 